Introduction The current gold standard assessment of diaphragm contractility involves invasive measures of transdiaphragmatic pressure (Pdi) (ATS/ERS 2002). Surface mechanomyography (sMMG) is a non-invasive measure of muscle fibre vibration during contraction. Sarlabous et al (ERJ 2015) reported a high correlation between diaphragm sMMG (sMMGdi) amplitude and inspiratory mouth pressure, a measure of global respiratory muscle function. To further validate the technique, the relationship between sMMGdi and Pdi was examined in this study, hypothesising that there would be a close relationship between sMMGdi and Pdi in healthy subjects.

Methods Pdi and sMMGdi (right lateral chest) were measured in 12 healthy subjects (6 male, age 33 (30-38) years, BMI $22.2(20.6-24.2) \mathrm{kg} / \mathrm{m} 2)$ during an increasing inspiratory threshold loading protocol. sMMGdi signals were analysed using fixed sample entropy (fSampEn). Mean and peak values, and the area under the curve of inspiratory Pdi and fSampEn sMMGdi, were calculated and compared.

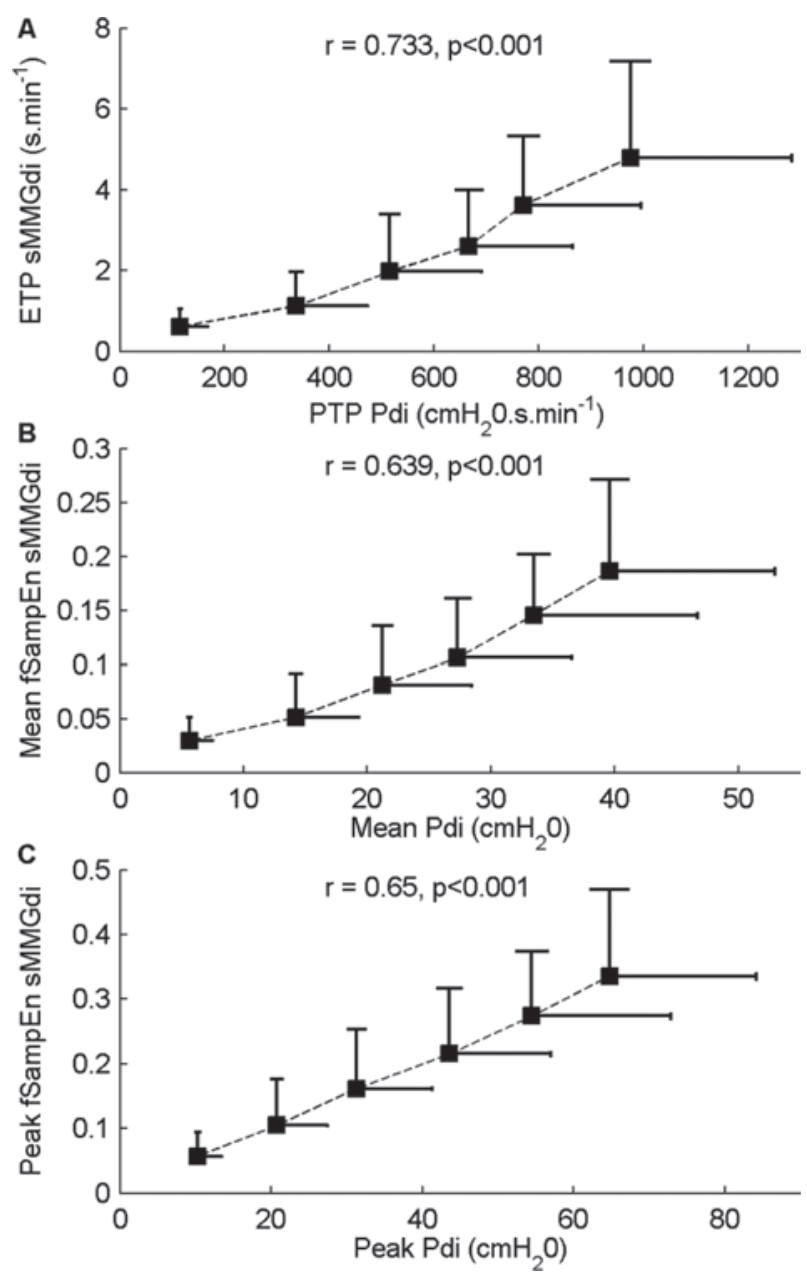

Abstract P136 Figure 1 Relationship between the area under the curve $(A)$, the mean value (B), and the peak value, and (C) of Pdi and fSampEn sMMGdi, during an increasing inspiratory threshold loading protocol. In each subfigure, the mean and standard deviation values of all healthy subjects are shown at rest and at $12 \%, 24 \%, 36 \%, 48 \%$, and $60 \%$ of maximum mouth inspiratory pressure. In subfigure $A$, the entropy-time product (ETP) arid the pressure-time product (PTP) represent the area under the fSampEn sMMGdi and Pdi curves, respectively, multiplied by the respiratory rate.
Results Strong correlations between the non-invasive sMMGdi and the invasive Pdi measures were observed for all three parameters (figure 1).

Conclusion The strong correlation between measures of sMMGdi and Pdi in healthy subjects suggests that sMMGdi could provide a clinically applicable noninvasive index of respiratory muscle contractility in patients with respiratory muscle weakness for diagnosis and monitoring.

\section{P137 19 F-MRI OF INHALED PERFLUOROPROPANE GAS: A NOVEL APPROACH TO VENTILATION IMAGING}

${ }^{1} \mathrm{~B}$ Pippard, ${ }^{1} \mathrm{M}$ Neal, ${ }^{2} \mathrm{P}$ Dutta, ${ }^{2} \mathrm{AJ}$ Simpson, ${ }^{1} \mathrm{P}$ Thelwall. ${ }^{1}$ Newcastle Magnetic Resonance Centre, Newcastle University, Newcastle upon Tyne, UK; ${ }^{2}$ Institute of Cellular Medicine, Newcastle University, Newcastle upon Tyne, UK

\subsection{6/thoraxjnl-2017-210983.279}

Introduction Current measures of pulmonary ventilation are beset by limitations. For example, spirometry provides no regional or anatomical information regarding lung function, while both CT and V/Q scans incur ionising radiation doses, restricting serial use. Hyperpolarised gas MRI (e.g., using ${ }^{3} \mathrm{He}$ and ${ }^{129} \mathrm{Xe}$ ) enables direct assessment of airway structure and function without recourse to ionising radiation, but is confined to centres with access to specialised hyperpolarising equipment and expertise. A novel approach, involving ${ }^{19} \mathrm{~F}-\mathrm{MRI}$ of inhaled perfluoropropane gas, has recently been described in humans, ${ }^{1,2}$ offering an alternative to hyperpolarisation with scope for translation to clinical practice.

Aim We assessed the feasibility of using inhaled perfluoropropane gas to image pulmonary ventilation properties in a group of healthy volunteers.

Methods 17 participants (10 M, 7 F; aged 21-52) provided written informed consent, and were screened for normal lung function using standard spirometry. Participants were invited to attend two MRI scanning sessions, during which they inhaled a $79 \%$ perfluoropropane $/ 21 \%$ oxygen gas mixture on up to three occasions. Gas inhalations lasted $<1 \mathrm{~min}$, typically involving 3-5 deep breaths followed by breath-hold. MRI scans of inhaled perfluoropropane were acquired using a Philips Achieva 3T scanner and a designated receiver coil tuned to ${ }^{19} \mathrm{~F}$ frequency. Heart rate and oxygen saturations were monitored throughout.

Results In total, 94 gas inhalations were performed across 17 participants. Ventilation images were obtained within a single breath-hold, demonstrating homogeneous gas distribution throughout the lungs (figure 1). Inhalation of the gas mixture was well tolerated with no significant adverse events, other than a transient (seconds) fall in $\mathrm{SpO} 2$ (89\%) following one breath-hold. This resolved spontaneously, and was not considered clinically significant.

Conclusions ${ }^{19}$ F-MRI of inhaled perfluoropropane gas represents a novel approach to ventilation imaging, with potential for high quality image acquisition within a single breath-hold. Crucially, this technique can be implemented on MRI scanners with considerably less additional hardware than required for hyperpolarised gas MRI. Ongoing optimisation of scan protocols will enable further improvements in both spatial and temporal resolution, providing a platform for future clinical application. 

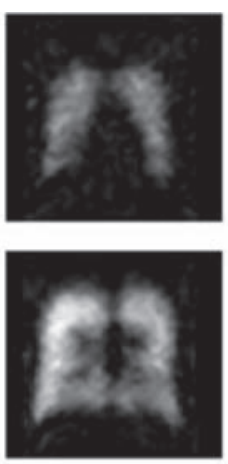
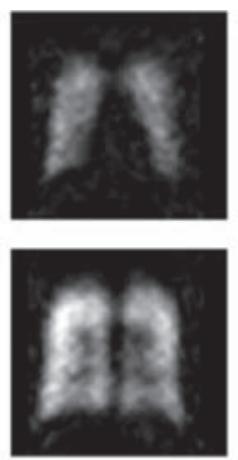
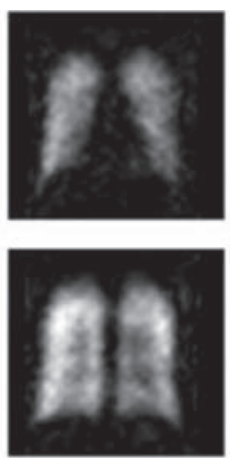
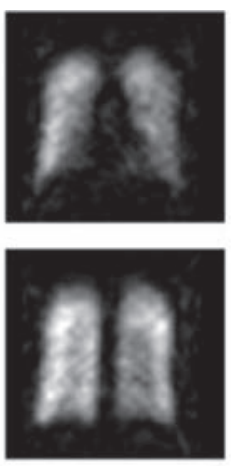
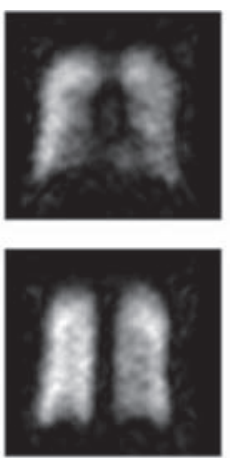
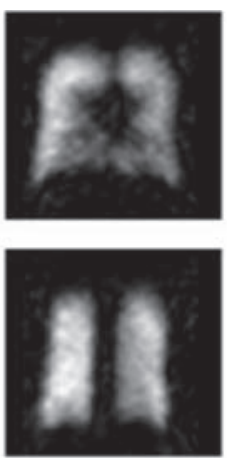

Abstract P137 Figure 1 Example 19F images (coronal views) Acquired during an 18s breath-hold after 3 deep gas inhalations.

\section{REFERENCES}

1. Couch MJ, et al. Radiology 2013;269:903-909.

2. Halaweish AF, et al. Chest 2013;144:1300-1310.

\section{P138 KINETICS OF INTRATHORACIC PRESSURE CHANGE FOLLOWING ADMINISTRATION OF CPAP}

MCP Apps, E Walsted, M Pavitt, L Swanton, A Lewis, S Buttery, J Garner, N Hopkinson, M Polkey, J Hull. Royal Brompton and Harefield NHS Trust, London, UK

\subsection{6/thoraxjnl-2017-210983.280}

Introduction An understanding of the changes in intra-thoracic pressure in response to application of Continuous Positive Airway Pressure (CPAP) is important in the study of thoracic and ventilator mechanics and device tolerability. It is unclear how quickly intra-thoracic pressure, measured directly with balloon catheters, responds to a change in CPAP. The aim of this study was to evaluate the kinetics of pressure stabilisation in healthy subjects.

Methods Mouth pressure (Pmo) was measured directly at the facemask of a NIPPY3 CPAP system, oesophageal pressure (Poes) and gastric (Pga) pressures were measured with balloon catheters in healthy subjects $(n=7)$, seated at rest, with $10 \mathrm{~min}$ spontaneous ventilation followed by $20 \mathrm{~min}$ at CPAP of $5 \mathrm{~cm} /$ $\mathrm{H}_{2} 0$, then $20 \mathrm{~min}$ at $\mathrm{CPAP} 10 \mathrm{~cm} / \mathrm{H}_{2} 0$, then $10 \mathrm{~min}$ no CPAP.

Results Pmo was lower than the setting for CPAP on the NIPPY3 machine; for $\mathrm{CPAP}=5 \mathrm{~cm} / \mathrm{H}_{2} 0$ mean $\mathrm{Pmo}=4.67 \mathrm{~cm} /$ $\mathrm{H}_{2} 0$, SD $0.29 \mathrm{~cm} / \mathrm{H}_{2} 0$; for $\mathrm{CPAP}=10 \mathrm{~cm} / \mathrm{H}_{2} 0$, mean Pmo $=9.09 \mathrm{~cm} / \mathrm{H}_{2} 0$, SD $0.3 \mathrm{~cm} / \mathrm{H}_{2} 0$. Poes with $5 \mathrm{~cm} / \mathrm{H}_{2} 0$ was higher than with no CPAP; $3.31 \mathrm{v} 0.13 \mathrm{~cm} / \mathrm{H}_{2} 0, \mathrm{p}<0.05$; with $10 \mathrm{~cm} / \mathrm{H}_{2} 0,5.16 \mathrm{v} 3.31 \mathrm{~cm} / \mathrm{H}_{2} 0, \mathrm{p}<0.05$; with $\mathrm{CPAP}$ back to $0 \mathrm{~cm} / \mathrm{H}_{2} 0,5.18 \mathrm{v} 1.1 \mathrm{~cm} / \mathrm{H}_{2} 0, \mathrm{p}<0.05$. There was a wide variability of gastric pressures both with and without CPAP; no significant changes in Pga with CPAP. Stabilisation of Pmo and Poes pressures after CPAP settings were changed occurred within $2 \mathrm{~min}$ for change in CPAP from $0-5 \mathrm{~cm} / \mathrm{H}_{2} 0$, 5-10 $\mathrm{cm} / \mathrm{H}_{2} \mathrm{O}$, and $10-0 \mathrm{~cm} / \mathrm{H}_{2} \mathrm{O}$ with Pmo maximum time to stabilise $80 \mathrm{~s}$, Poes maximum time to stabilise $86 \mathrm{~s}$. Pga stabilisation took longer; for CPAP setting change $0-5 \mathrm{~cm} / \mathrm{H}_{2} 0$, time to stability for Pga was $111-470 \mathrm{~s}$; for CPAP $5-10 \mathrm{~cm} /$ $\mathrm{H}_{2} 0$, 46-183 s; for CPAP setting change 10-0 $\mathrm{cm} / \mathrm{H}_{2} 0,37-$ $135 \mathrm{~s}$.

Conclusions In healthy subjects the kinetics of thoracic pressure stabilisation, following application of CPAP, is highly variable. Gastric pressure takes longer to stabilise and varies more than Pmo and Poes. This may reflect variation in diaphragm tonicity, gastric contraction or abdominal wall tone. These variable time constraints need considering when evaluating CPAP intervention. Subject variability in gastric pressure may contribute to reduced tolerability in some individuals and requires further study.

\section{P139 A RANDOMISED COMPARATIVE STUDY OF COUGH PEAK EXPIRATORY FLOW (CPEF) USING FULL FACE MASK VS MOUTHPIECE INTERFACES IN HEALTHY SUBJECTS}

A Mearns, F Subhan, L Roberts. University of Plymouth, Plymouth, UK

\subsection{6/thoraxjnl-2017-210983.281}

Background Cough Peak Expiratory Flow (CPEF) is a respiratory muscle function test designed to assess the ability to clear airway secretions adequately. Present practice requires CPEF to be measured using a mouthpiece, which has proven problematic in patients the neuromuscular disease(s). The study aimed to determine the effectiveness of using a facemask vs mouthpiece in measuring CPEF.

Hypothesis CPEF measured via an Interurgical Anaesthetic Full-Face Mask will provide comparably similar (CI 0.95) Results to those obtained using a flanged mouthpiece.

Participant Population Healthy participants were recruited into the study through faculty newsletters, social media advertisements and random convenient sampling of network connexions. Participants were screened, following ethical approval, using a specifically-designed Pre-screening Medical Questionnaire (PSMQ) against an Inclusion criterion, before inclusion to the study.

Methods Testing procedure ensured standard spirometry position was adopted. The participant was asked to expire to residual volume (RV), followed by a rapid inhalation to total lung capacity (TLC) where a forceful cough manoeuvre was made. Procedure was repeated at least 3 times, with $45 \mathrm{~s}$ rest between attempts. A maximum of 8 attempts per interface was allowed, with a 10 min change-over period between interfaces. A students 2-sample t-test, Bland-Altman and regression analysis were employed to statistically analyse the data. Randomisation occurred using the exce RAND command on the sample ID's.

Results 60 healthy subjects were recruited, of which 58 participant's Results were deemed appropriate to study. The mean result of each interface was analysed to indicate no significant differences of CPEF measurements in healthy subjects (CI $95 \%, \mathrm{p}=0.971)$. There were no significant differences between Age and Gender (CI 95\%, Age $\mathrm{p}=0.453$, Gender $\mathrm{p}=0.902$ ) 